\title{
OWL: a code for the two-center shell model with spherical Woods-Saxon potentials
}

\author{
Alexis Diaz-Torres* \\ Department of Physics, University of Surrey, Guildford, GU2 7XH, UK
}

\begin{abstract}
A Fortran-90 code for solving the two-center nuclear shell model problem is presented. The model is based on two spherical Wood-Saxon potentials and the potential separable expansion method. It describes the single-particle motion in low-energy nuclear collisions, and is useful for characterizing a broad range of phenomena from fusion to nuclear molecular structures.

Keywords: Two-center shell model; Woods-Saxon potential; Potential separable expansion method; Adiabatic and diabatic single-particle states; Fusion
\end{abstract}

\footnotetext{
PROGRAM SUMMARY

Program Title: OWL

Licensing provisions: GPLv3

Programming language: Fortran-90

Nature of problem(approx. 50-250 words):

The two-center shell model (TCSM) is a basic microscopic model to describe the single-particle (sp) motion during a heavy ion collision near the Coulomb barrier. The sp energies and wave-functions are obtained solving the Schrödinger equation with a phenomenological two-center mean field based on two spherical WoodsSaxon (WS) potentials [1].

Solution method(approx. 50-250 words):

The potential separable expansion (PSE) method using two basis sets of harmonic oscillator functions is employed $[2,3]$. A method to ensure the volume conservation of the fusing system is used [1].

Additional comments including Restrictions and Unusual features (approx. 50-250
}

\footnotetext{
${ }^{*}$ Corresponding author.

E-mail address: a.diaztorres@surrey.ac.uk 
words):

The program is suited for two spherical WS potentials and provides bound sp states only.

[1] A. Diaz-Torres, W. Scheid, Nucl. Phys. A 757 (2005) 373-389.

[2] F.A. Gareev, M.Ch. Gizzatkulov, J. Revai, Nucl. Phys. A 326 (1977) 512-522.

[3] B. Gyarmati, A.T. Kruppa, J. Revai, Nucl. Phys. A 326 (1979) 119-128.

\section{Introduction}

The theoretical framework as well as the key techniques implemented in the present code are explained in Ref. [1]. The two-center problem is solved using two spherical WS potentials and the PSE method $[2,3]$. The WS potential parameters in a realistic calculation have to be adjusted so as to fit the experimental sp spectra around the Fermi level for the separated nuclei and for the spherical compound nucleus. For overlapping nuclei, these parameters can be interpolated as discussed in Ref. [1]. The present TCSM has the following advantages compared to the traditional one based on two harmonic oscillators [7]: (i) very asymmetric reactions can be described as those used to form the heaviest nuclei, (ii) the relative level positions in the colliding nuclei are correct, (iii) the two-center potential barrier between the nuclei is realistic [8], (iv) the Coulomb interaction for protons can be explicitly included, (v) the sp wave-functions have a correct asymptotic behaviour [2], and (vi) continuum states like Gamow resonances can be included [4], which is crucial for reactions of weakly bound nuclei. The PSE method has been used in conjunction with both the one-center problem $[5,6,9]$ and the two-center problem [10] for nuclear structure studies. The PSE method has also been combined with the diagonalization method [11, 12], and applied to low-energy collisions of spherical and deformed heavy ions [13, 14, 15].

Although the TCSM has a successful history since its introduction in heavy-ion physics by the Frankfurt school [17], there is no code publicly available at the moment. The present program aims to become the first public code, which can be useful for many applications in low-energy nuclear physics [16]. The formalism and methods are described in Section 2. In Section 3, the program and the input file are explained. The code is illustrated in Section 4 with the reaction ${ }^{16} \mathrm{O}+{ }^{40} \mathrm{Ca} \rightarrow{ }^{56} \mathrm{Ni}[1,8]$. 


\section{Formalism and methods}

The TCSM potential for neutrons can be constructed by the superposition of two shifted spherical WS potentials, which for protons also includes Coulomb potentials, $V_{\text {Coul }}^{s}$, with $s=1,2$. The Coulomb potential is taken to be that of a uniformly charged sphere with charge $Z_{s} e\left(Z_{s}\right.$ being the total charge of each nucleus) and the radius $R_{C}^{s}$. For instance, the neutron two-center potential reads as:

$$
V=\sum_{s=1}^{2} \exp \left(-i \mathbf{R}_{s} \hat{k}\right) V_{s} \exp \left(i \mathbf{R}_{s} \hat{k}\right),
$$

where $\hat{k}=\hbar^{-1} \hat{p}$ is the sp wave-number operator. The centers are located at $\mathbf{R}_{1}$ and $\mathbf{R}_{2}$, the relative coordinate is $\mathbf{R}=\mathbf{R}_{1}-\mathbf{R}_{2}$. The WS potentials, $V_{s}$, include a spin-orbit term:

$$
V_{s}(r)=-V_{0}^{s}\left[f^{s}(r)-\frac{\kappa_{s}}{r} \frac{d f_{s o}^{s}(r)}{d r}(\mathbf{l} \cdot \mathbf{s})\right]
$$

where $f^{s}$ and $f_{s o}^{s}$ are the same function, but with different parameters, i.e., $f_{(s o)}^{s}=\left\{1+\exp \left[\left(r-r_{0(s o)}^{s} A_{s}^{1 / 3}\right) / a_{0(s o)}^{s}\right]\right\}^{-1}, A_{s}$ being the mass number of each nucleus. $\kappa_{s}$ refers to the strength of the spin-orbit interaction.

Each potential (2) is approximately represented $\left(V_{s e p}^{s}\right)$ within a truncated sp harmonic oscillator basis, with the spin-angular part coupled to the total angular momentum $j$ with projection $m$ [1]. This basis adopts essentially the same mathematical form in momentum and coordinate representations. In the coordinate representation (spherical coordinates), the radial part of the basis can be written as $\langle r \mid n l\rangle=h_{o s c}^{-3 / 2} \phi_{n l}(x)$, where $h_{o s c}$ is the oscillator length and the adimensional $x=r / h_{\text {osc }}$. The oscillator length associated with each basis set is calculated with the expression $h_{o s c}^{s}=0.84 \cdot r_{0}^{s} \cdot A_{s}^{1 / 6} \mathrm{fm}$ [1]. In the momentum representation, $\langle k \mid n l\rangle \equiv g_{n l}(k)=(-1)^{n} i^{l} h_{\text {osc }}^{3 / 2} \phi_{n l}(\xi)$, being the adimensional $\xi=h_{o s c} k$. The function $\phi_{n l}$ is the reduced oscillator function:

$$
\phi_{n l}(\xi)=\sqrt{\frac{2 n !}{\Gamma(n+l+3 / 2)}} \xi^{l} e^{-\xi^{2} / 2} L_{n}^{l+1 / 2}\left(\xi^{2}\right),
$$

where $L_{n}^{l+1 / 2}\left(\xi^{2}\right)$ is a Laguerre polynomial.

The approximate potentials, $V_{s e p}^{s}$, read: 


$$
V_{s} \approx V_{s e p}^{s}=\sum_{\nu, \mu=1}^{N}|s \nu\rangle V_{\nu \mu}^{s}\langle s \mu|,
$$

where $\nu, \mu$ denote the set of quantum numbers $\{n l j m\}$ of the spherical harmonic-oscillator functions. The number $N$ of basis states included in the expansion (4) is defined by $l_{\max }$ (number of partial waves in which the potential acts) and $n_{\max }$ (the number of separable terms in each partial wave). The values of $l_{\max }$ and $n_{\max }$ are determined by the convergence of the sp energies which is accelerated using the Lanczos $\sigma$-factors that remove the Gibbs phenomenon [11].

The two-center problem for bound sp states is solved in the momentum representation by means of a set of linear algebraic equations, derived from the formal solution of the Schrödinger equation with the potentials (1) and (4), as explained in Ref. [1]. This set of algebraic equations is:

$$
\sum_{\mu^{\prime}=1}^{N} \sum_{s^{\prime}=1}^{2}\left[\delta_{s s^{\prime}} \delta_{\mu \mu^{\prime}}-\sum_{\nu=1}^{N}\left\langle s \mu\left|G_{0}(E) e^{i\left(\mathbf{R}_{s}-\mathbf{R}_{s^{\prime}}\right) \hat{k}}\right| s^{\prime} \nu\right\rangle V_{\nu \mu^{\prime}}^{s^{\prime}}\right] A_{s^{\prime} \mu^{\prime}}=0
$$

where $G_{0}(E)=\left(E-\hbar^{2} \hat{k}^{2} / 2 m_{0}\right)^{-1}$ is the Green operator for the free sp motion. The energy eigenvalues $E$, which appear as a parameter in the matrix elements containing $G_{0}$, are determined from the condition that the determinant of the system (5) vanishes. With the eigenvalues $E$, the eigenvectors $|\varphi\rangle$ are obtained solving the system (5) for the coefficients $A_{s \mu}$ and requiring the normalization of the states $|\varphi\rangle$. These adiabatic molecular sp states read as:

$$
|\varphi\rangle=G_{0}(E) \sum_{\nu, \mu=1}^{N} \sum_{s=1}^{2} V_{\nu \mu}^{s} A_{s \mu} e^{-i \mathbf{R}_{s} \hat{k}}|s \nu\rangle .
$$

The molecular sp states are characterized by the projection of the total angular momentum $j$ along the internuclear axis, $\Omega$, which is a good quantum number due to the axial symmetry of the two-center potential (1). In the body-fixed reference frame $\left(\mathbf{R}_{1}=\mathbf{R}\right.$ and $\left.\mathbf{R}_{2}=0\right)$, the matrix elements containing $G_{0}$ are as follows $\left[\hat{j}=(2 j+1)^{1 / 2}\right]$ :

$$
s=s^{\prime}:\left\langle n l j \Omega\left|G_{0}(E)\right| n^{\prime} l^{\prime} j^{\prime} \Omega^{\prime}\right\rangle=\delta_{l l^{\prime}} \delta_{j j^{\prime}} \delta_{\Omega \Omega^{\prime}}\left\langle n l\left|G_{0}(E)\right| n^{\prime} l^{\prime}\right\rangle,
$$




$$
\begin{array}{r}
s \neq s^{\prime}:\left\langle n l j \Omega\left|G_{0}(E) e^{i \mathbf{R}_{s s^{\prime}} \hat{k}}\right| n^{\prime} l^{\prime} j^{\prime} \Omega^{\prime}\right\rangle=\delta_{\Omega \Omega^{\prime}}(-1)^{\Omega+1 / 2} \\
\times \sum_{L} i^{L+l-l^{\prime}} Q_{s s^{\prime}} \hat{l} \hat{j} \hat{l}^{\prime} \hat{j}^{\prime}\left(l 0 l^{\prime} 0 \mid L 0\right)\left(j \Omega j^{\prime}-\Omega \mid L 0\right) \\
\times\left\{\begin{array}{ccc}
L & l^{\prime} & l \\
\frac{1}{2} & j & j^{\prime}
\end{array}\right\}\left\langle n l\left|G_{0}(E) j_{L}(k R)\right| n^{\prime} l^{\prime}\right\rangle
\end{array}
$$

where $j_{L}$ refers to the spherical Bessel function, and

$$
\begin{gathered}
Q_{s s^{\prime}}=\left\{\begin{array}{l}
1, \quad s s^{\prime}=12, \\
(-1)^{L}, \quad s s^{\prime}=21,
\end{array}\right. \\
\left\langle n l\left|G_{0}(E)\right| n^{\prime} l^{\prime}\right\rangle=-\frac{2 m_{0}}{\hbar^{2}} \int_{0}^{\infty} \frac{g_{n l}^{*}(k) g_{n^{\prime} l^{\prime}}(k)}{k^{2}+\gamma^{2}} k^{2} d k, \gamma^{2}=-\frac{2 m_{0} E}{\hbar^{2}}, \\
\left\langle n l\left|G_{0}(E) j_{L}(k R)\right| n^{\prime} l^{\prime}\right\rangle=-\frac{2 m_{0}}{\hbar^{2}} \int_{0}^{\infty} \frac{g_{n l}^{*}(k) g_{n^{\prime} l^{\prime}}(k) j_{L}(k R)}{k^{2}+\gamma^{2}} k^{2} d k .
\end{gathered}
$$

The calculation of the matrix elements (7)-(11) is explained in Ref. [1].

Adiabatic sp states diagonalize the two-center sp Hamiltonian, whereas the diabatic states minimize the strong dynamical non-adiabatic radial coupling at an avoided crossing between two adiabatic sp levels with the same symmetry [1]. Diabatic states with the same quantum numbers can cross each other because they are not solutions of an eigenvalue problem. Appendix A in Ref. [1] provides the radial non-adiabatic coupling matrix elements, $\left\langle\right.$ ind $x \Omega|\partial / \partial R|$ ind $\left.x^{\prime} \Omega\right\rangle$, where indx distinguishes different states with the same $\Omega$. Diagonalizing these coupling at an avoided crossing, we obtain the unitary transformation matrix, $S_{i, j}^{\Omega}$, between the adiabatic and diabatic states, $i, j$ referring to the values of $i n d x$. The diabatic sp energies and wave functions are:

$$
\begin{gathered}
|i \Omega\rangle_{\text {diab }}=\sum_{j} S_{i j}^{\Omega}|j \Omega\rangle_{a d i a b} \\
E_{i \Omega}^{\text {diab }}=\sum_{j}\left|S_{i j}^{\Omega}\right|^{2} E_{j \Omega}^{a d i a b} .
\end{gathered}
$$




\subsection{Volume conservation}

To describe the fusion process, the WS potential parameters are interpolated between their values for the separated nuclei and the spherical compound nucleus, making use of the condition of volume conservation for an equipotential surface that determines the nuclear shapes [1]. The parameters of both the asymptotic WS potentials and the potential of the spherical compound nucleus, including the spin-orbit term, reproduce either the experimental single-particle energy levels around the corresponding Fermi surfaces or the experimental single-particle separation energies.

The parameters $\mathcal{P} \equiv\left\{V_{0}^{s}, r_{0(s o)}^{s}, a_{0(s o)}^{s}, \kappa_{s}, R_{C}^{s}, Z_{s}\right\}$ are interpolated by means of the unknown function $y(R)$ :

$$
\mathcal{P}(R)=\mathcal{P}(R=0)[1-y(R)]+\mathcal{P}(R \rightarrow \infty) y(R),
$$

where $y(R)$ satisfies the boundary conditions $y(R=0)=0$ and $y(R \rightarrow \infty)=$ 1. The function $y(R) \in[0,1]$ is numerically calculated by inserting (14) into the volume conservation condition:

$$
\int d v \Theta\left(\Sigma_{0}-\Sigma\right)=v_{0}
$$

where $\Theta$ is the Heaviside step function, $\Sigma_{0}$ is the fixed equipotential surface corresponding to the Fermi level of the spherical compound nucleus, and $v_{0}$ is the volume enclosed by the equipotential surface $\Sigma_{0}$. The parameters $\mathcal{P}(R \rightarrow \infty)$ can be unambiguously found, but this is not the case for the individual fragment potentials at $R=0$ where only the sp spectra of the compound nucleus are known. At $R=0$, we consider that $r_{0(s o)}^{s}, a_{0(s o)}^{s}, \kappa_{s}$ and $R_{C}^{s}$ are the same as those of the compound nucleus, but $V_{0}^{1}+V_{0}^{2}=V_{0}^{C N}$ and $Z_{1}+Z_{2}=Z_{C N}$. A new parameter $\alpha=V_{0}^{1} / V_{0}^{C N}=Z_{1} / Z_{C N}$ is introduced, which determines the values of $V_{0}^{s}$ and $Z_{s}$ at $R=0$. This $\alpha \in(0,1)$ is chosen in such a way that the splitting of the sp energy levels at small $R$ resembles the Nilsson model, as demonstrated in Fig. 2 of Ref. [1]. We found that a realistic value of $\alpha$ is about 0.8 . For comparison between the Nilsson model and the present model, the energies in Fig. 2 of Ref. [1] are shown relative to the first Nilsson-level and in units of $\hbar \omega_{0}=41 \cdot A^{-1 / 3} \mathrm{MeV}$ with $A=56$. 


\section{Computer program and input file}

\subsection{Structure of the code}

Solving the system of equations (5) - considering the volume conservation condition (15) - is the central aspect of the present code. The code has a main program and sixteen modules. The main program Two Center directs the input to be read, the problem to be solved, and details of the calculation to be written in output files. The modules are kinds, global data, oscillator, anglib, nrutil, sep potential, kinetic matrix elements, wave functions, energy partition, single particle energies, wf phases, diabaticity, universal potentials, interpolation integrals, output spectra, volume conservation condition and input data. The program requires an additional file (auxiliaryalgebra.f) which contains subroutines and their dependencies from LINPACK and NETLIB.

The main program Two Center calls the module input data first, in which the subroutine input reads the input file described below. Thereafter, a number of angular momentum coupling coefficients as well as gamma and factorial functions are determined using the modules anglib and oscillator. Depending on the value of different output control variables, details of the calculation can be written into output files. The output file TRACKING CALCULATIONS contains information on the evolution of the calculations. The calculations are sequentially carried out for neutrons ( $\mathrm{ISO}=-1$ ) with specific magnetic substates characterized by the variable OMEG, followed by the same calculations for protons $(\mathrm{ISO}=1)$. Having fixed the ISO and OMEG values, the main program calls the subroutine global integrals in the module interpolation integrals to evaluate integrals that will be interpolated later on. The two-center Schrödinger equation is then solved for different separations between the WS potentials. The number of internuclear distances increases by a factor of three, if the diabatic sp spectra is calculated as well, which happens when the input variable ADIABATICITY=0. At a fixed internuclear distance, the main program calls various subroutines: potential matrix in the module sep potentials for the calculation of the potential matrices, global integrals and D2_kineticmatrices_E2 in the module interpolation integrals, kinetic matrix in the module kinetic matrix elements, all of them for making the calculation of sp states more efficient. The adiabatic and diabatic sp states are calculated with the subroutines energies and diabatic states in the modules single particle energies and diabaticity, respectively. Finally, the main program calls the subroutine spectra in the module output spectra for writing the adiabatic and diabatic spectra into different output files. 
Module kinds. This defines the kind type parameter for real values.

Module global data. This defines global variables used in different modules.

Module oscillator. Subroutines for the reduced oscillator wave-functions in the coordinate and momentum representations are given here. It also contains some special functions such as the gamma, the Bessel and the spherical functions.

Module anglib. It contains a library of angular momentum coupling coefficient subroutines for those coefficients in expression (8).

Module nrutil. In this module, several subroutines from Numerical Recipies are included, which perform various tasks such as matrix diagonalization, Gauss-Legendre quadrature, spline interpolation and singular value decomposition of a matrix.

Module sep potential. In this module, the subroutine potential matrix calculates the potential matrices, $V_{\nu \mu}^{s}$, using the reduced oscillator wavefunctions in coordinate representation. It includes the Lanczos $\sigma$-factors [11] to accelerate the convergence with respect to the maximal number of nodes of the oscillator functions.

Module kinetic matrix elements. The subroutine kinetic matrix calculates matrix elements of the free sp Green operator, $G_{0}(E)$, making use of analytical and recurrence relations $[1,4,9]$ as well as spline interpolation in the subroutine kinetic matrix interp.

Module interpolation integrals. Here, the subroutines global integrals and D2_kineticmatrices_E2 provide a large number of matrix elements of the free sp Green operator for spline interpolation.

Module wave functions. Here, the subroutine wave function coef provides coefficients, $A_{\nu \mu}^{s}$, of the sp wave-functions in the momentum representation.

Module energy partition. This module includes the subroutine minimum energy, which tracks the sign of the determinant. When the determinant changes its sign, the subroutine ZBRENT determines the sp energies, while the subroutine parabolic approach separates very close sp energies.

Module single particle energies. Here, the subroutine energies calculates the determinant evaluated over a dense grid of energies, passing the determinant value on to the subroutine minimum energy in the module energy partition. The determinant is calculated using the subroutines zgefa and zgedi from LINPACK.

Module wf phases. This module contains the subroutine phase correction, which determines the correct phases of the wave-function coefficients 
by minimizing the radial non-adiabatic couplings. It is important for the calculation of diabatic states [1].

Module diabaticity. The subroutine diabatic states provides diabatic states by diagonalizing the radial non-adiabatic coupling matrix [1].

Module output spectra. In this module, the subroutine spectra writes the adiabatic and diabatic sp energies of specific magnetic substates into output files.

Module volume conservation condition. Here, the subroutine fvalues calls the subroutine shape volume, determining a function based on the volume conservation condition (e.g., see Fig. 1 in Ref. [1]). This function is useful for interpolating the WS parameters for overlapping nuclei (14). The subroutine shape volume calculates the volume enclosed by an equipotential shape (15) using the subroutine DCUHRE from NETLIB for multidimensional integration.

Module universal potentials. In this module, the subroutine potential parametrization determines global WS parameters to be used in the calculations, provided the input variable PARAMETERS POTENTIAL $\neq 0$ : $(1)$ Bohr and Mottelson, (2) Soloviev, and (3) Lund.

Module input data. The subroutine input reads the input file, and calls the subroutines potential parametrization (if PARAMETERS POTENTIAL $\neq 0)$ and fvalues in the modules universal potentials and volume conservation condition, respectively.

\subsection{Input file}

Figure 1 shows the input file of the program. At the bottom of the figure, there is the namelist of the input variables appearing in the code, which correspond to some physics variables explained in Sect. 2. Most of the lines are self-explanatory, while some variables have already been mentioned in the description of the program. Line 16 refers to two constants for neutrons and protons, which determine the value of the depth of the individual potentials at zero separation (i.e., the $\alpha$ parameter, which is illustrated in Fig. 2 of Ref. [1]). Line 18 provides the Fermi levels of the spherical compound nucleus for neutrons and protons, which are determined by the potential parameters given in lines 11-15. These Fermi levels are used to calculate the volume enclosed by the corresponding equipotential shapes, which is preserved for overlapping nuclei. Line 20 allows the user to run the code for a fixed internuclear distance (R_fixed), provided the variable FIXED_DISTANCE=1. 


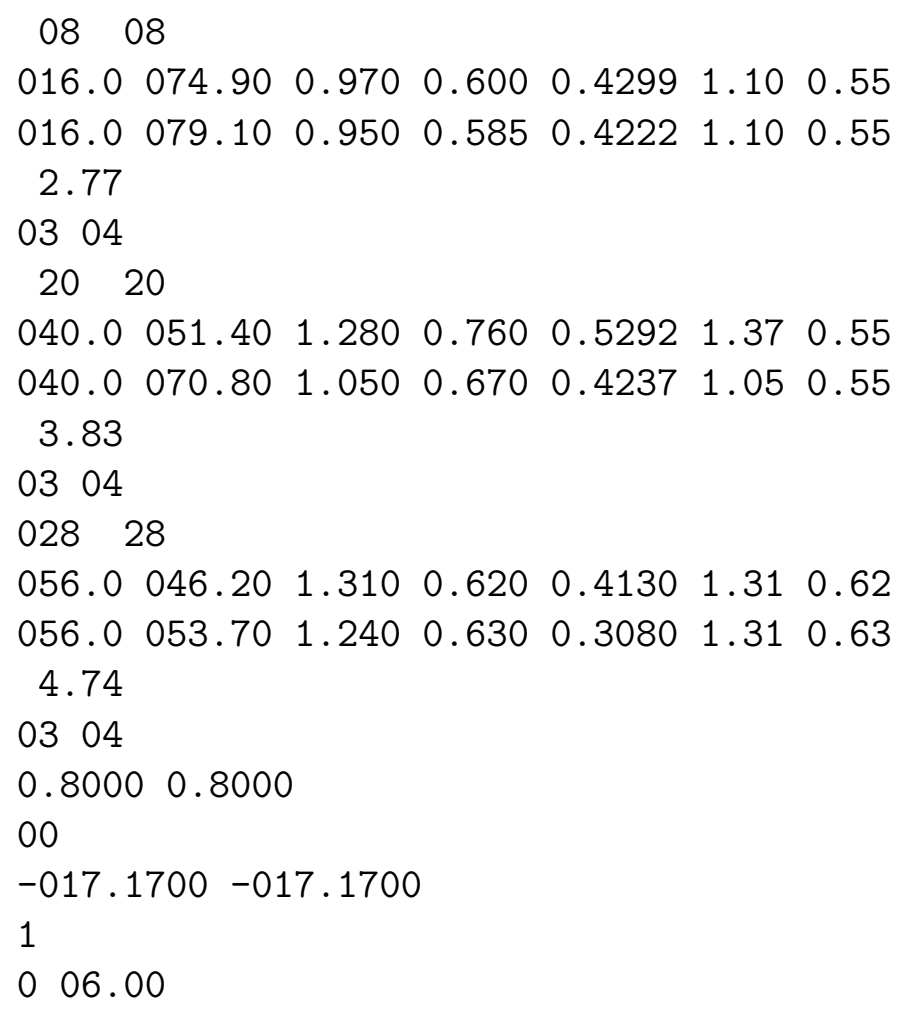

LINE 1-5: Wood_Saxon potential 1: A, V0,r0,a0,kappa,rs, as Wood-Saxon potential parameters: mass number, depth, radius constant, difussivity; the same for spin-orbit part. $\mathrm{V}=-\mathrm{V} 0\left[\mathrm{f}(\mathrm{r})-\operatorname{kappa}\left(\mathrm{df}_{-} \mathrm{s}(\mathrm{r}) / \mathrm{rdr}\right) 1 * \mathrm{~s}\right]$ $f(r)=1 /[1+\exp ((r-r 0 * A * *(1 / 3)) / a 0)]$

LINE 6-10: Wood_Saxon potential 2:

FMT $=$ ' $(I 3,1 \mathrm{X}, \mathrm{I3})^{\prime}$ ') NEUT2, PROT2 


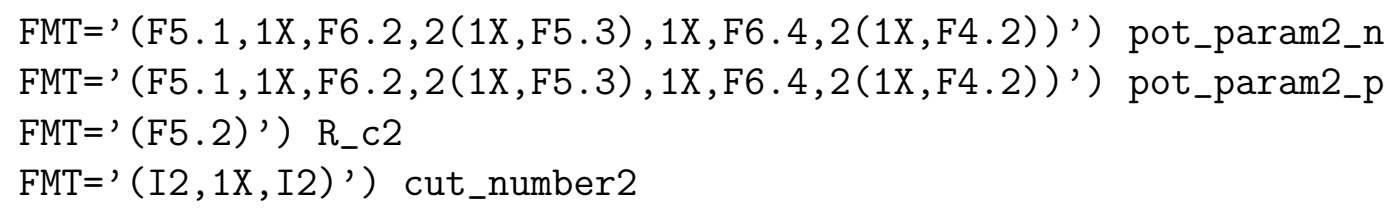

LINE 11-15: Wood_Saxon potential of the spherical CN:

FMT $=$ ' $\left.(I 3,1 \mathrm{X}, \mathrm{I3})^{\prime}\right)$ NEUT3, PROT3

$\mathrm{FMT}=$ ' $\left.(\mathrm{F} 5.1,1 \mathrm{X}, \mathrm{F} 6.2,2(1 \mathrm{X}, \mathrm{F} 5.3), 1 \mathrm{X}, \mathrm{F} 6.4,2(1 \mathrm{X}, \mathrm{F} 4.2))^{\prime}\right)$ pot_param3_n

$\mathrm{FMT}=$ ' $(\mathrm{F} 5.1,1 \mathrm{X}, \mathrm{F} 6.2,2(1 \mathrm{X}, \mathrm{F} 5.3), 1 \mathrm{X}, \mathrm{F} 6.4,2(1 \mathrm{X}, \mathrm{F} 4.2))^{\prime}$ ') pot_param3_p

$\mathrm{FMT}=$ ' $\left.(\mathrm{F} 5.2)^{\prime}\right) \mathrm{R}_{-} \mathrm{c3}$

FMT $=$ ' $\left.(I 2,1 \mathrm{X}, \mathrm{I} 2)^{\prime}\right)$ cut_number3

LINE 16: Depth of the fragments potential at $R=0 \mathrm{fm}$ :

FMT =' (F6.4,1X,F6.4)') gam_n,gam_p

LINE 17: For selecting either the above potentials or global potentials:

00-above potentials, 01-Bohr\&Mottelson, 02- Soloviev, 03-Lund

FMT $=$ ' $(\mathrm{I} 2,2 \mathrm{X}, \mathrm{I} 2)^{\prime}$ ') PARAMETERS_POTENTIAL

LINE 18: Neutron and proton Fermi levels of the spherical CN.

FMT =' (F9.4,1X,F9.4)') EPS_n,EPS_p

LINE 19: ADIABATICITY of the single-particle motion:

1- adiabatic, 0- diabatic case is included.

LINE 20: For fixing a distance between the two potentials:

FIXED_DISTANCE ( 1 -activated, 0-disactivated)

R_fixed is the fixed distance ( $\mathrm{fm}$ )

FMT =' (I1,1X,F5.2)' ) FIXED_DISTANCE, R_fixed

Fig. 1. Input file for OWL code (owl.inp).

\section{Test run}

Figure 2 shows the correlation diagrams of the adiabatic energy levels for (a) neutrons and (b) protons, which result from the input file in Fig. 1. These energies are stored in the output files SPECTRUM_ADIAB_OMEG for every $\Omega$ value. Different magnetic substates characterized by the quantum 
number $\Omega$ are represented by different types of line. As expected, the singleparticle levels are highly polarized at intermediate separations, the proton levels being shifted up relative to the neutron levels because of the Coulomb interaction.

Figure 3 presents the occupation probability $\left(\left|A_{s \mu}\right|^{2}\right)$ of the two WS potentials for a neutron orbit with $\Omega=5 / 2$, which is asymptotically localized in the ${ }^{40} \mathrm{Ca}$ potential with energy of $-18.58 \mathrm{MeV}$. As expected, this state gradually becomes delocalized as the ${ }^{16} \mathrm{O}$ and ${ }^{40} \mathrm{Ca}$ nuclei come into contact. The geometrical contact radius is about $7.13 \mathrm{fm}$. The occupation probabilities can be found in the output file TRACKING_CALCULATIONS.

Table 1: Convergence relative to $n_{\max }$ of neutron energies with both $\Omega=1 / 2$ (columns 2 and 3) and $\Omega=5 / 2$ (columns 4 and 5 ), at the internuclear distance $R=4 \mathrm{fm}$.

\begin{tabular}{ccccc}
\hline \hline$n_{\max }$ & $E_{1}^{1 / 2}(\mathrm{MeV})$ & $E_{2}^{1 / 2}(\mathrm{MeV})$ & $E_{1}^{5 / 2}(\mathrm{MeV})$ & $E_{2}^{5 / 2}(\mathrm{MeV})$ \\
\hline 2 & -64.974 & -2.664 & -28.178 & -1.539 \\
3 & -64.941 & -2.232 & -28.790 & -1.436 \\
4 & -64.943 & -1.989 & -28.780 & -1.395 \\
5 & -64.945 & -1.842 & -28.792 & -1.363 \\
6 & $\mathbf{- 6 4 . 9 4 6}$ & -1.865 & -28.791 & $\mathbf{- 1 . 3 5 6}$ \\
7 & -64.946 & -1.874 & $\mathbf{- 2 8 . 7 9 0}$ & -1.356 \\
8 & & -1.867 & -28.790 & \\
9 & & -1.866 & & \\
10 & & -1.864 & & \\
11 & & $\mathbf{- 1 . 8 6 3}$ & \\
12 & & -1.863 &
\end{tabular}

Table 1 presents the convergence with respect to $n_{\max }$ of neutron energies with both $\Omega=1 / 2$ and $\Omega=5 / 2$ at $R=4 \mathrm{fm}$ in Fig. 2(a). Although convergence is always achieved, it is observed that the required number of separable terms for weakly bound states with small $\Omega$ (column 3 ) is significantly larger than that for either strongly bound states (columns 1 and 4) or weakly bound states with large $\Omega$ (column 5 ). 

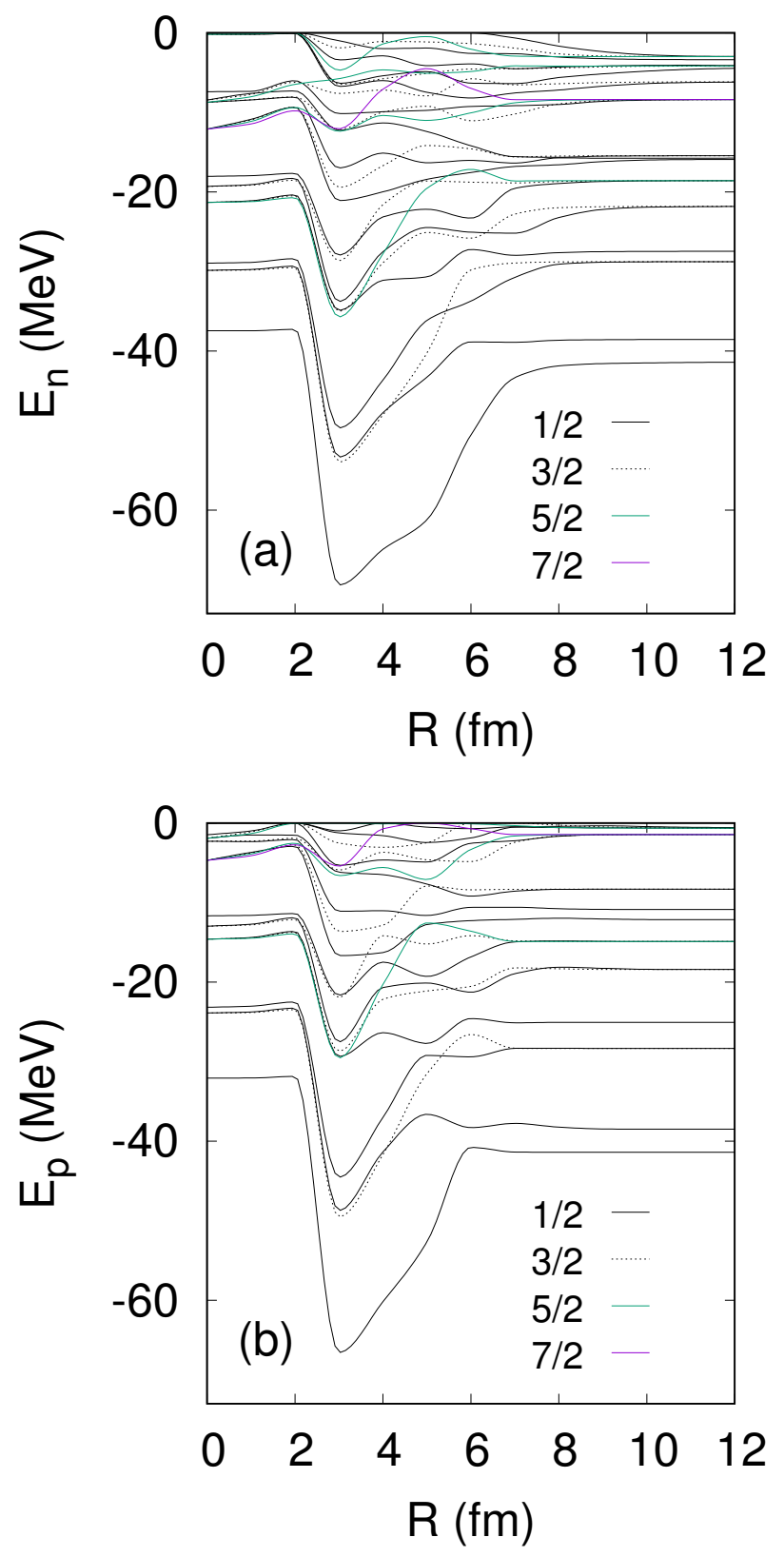

Figure 2: (Color online) (a) Adiabatic neutron energies as a function of the internuclear distance for the ${ }^{16} \mathrm{O}+{ }^{40} \mathrm{Ca} \rightarrow{ }^{56} \mathrm{Ni}$ collision. Different types of line denote different $\Omega$ values of the projection of the total angular momentum along the internuclear axis. (b) The same for protons. 


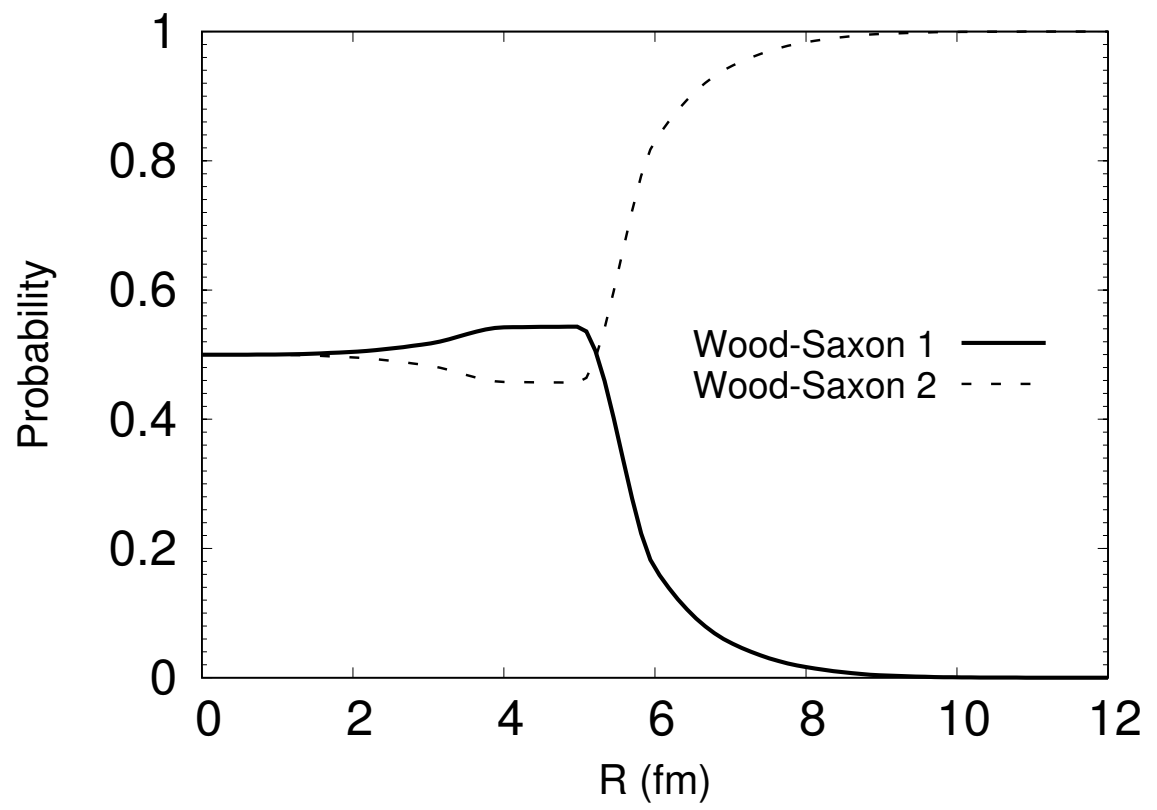

Figure 3: Occupation probability of the WS potentials for a neutron state with $\Omega=$ $5 / 2$ in Fig. 2. This state asymptotically belongs to ${ }^{40} \mathrm{Ca}$ with energy of $-18.58 \mathrm{MeV}$. Delocalization of this state happens when the ${ }^{16} \mathrm{O}$ and ${ }^{40} \mathrm{Ca}$ nuclei come into contact. 
Acknowledgement The work was supported by the UK Science and Technology Facilities Council (STFC) New Applicant Grant No. ST/P00671X/1.

[1] A. Diaz-Torres, W. Scheid, Nucl. Phys. A 757 (2005) 373-389.

[2] F.A. Gareev, M.Ch. Gizzatkulov, J. Revai, Nucl. Phys. A 326 (1977) 512-522.

[3] B. Gyarmati, A.T. Kruppa, J. Revai, Nucl. Phys. A 326 (1979) 119-128.

[4] B. Milek, R. Reif, Nucl. Phys. A 458 (1986) 354-380.

[5] F.A. Gareev, S.N. Ershov, J. Revai, J. Bang, B.S. Nilsson, Phys. Scr. 19 (1979) 509-515.

[6] B. Gyarmati, A.T. Kruppa, Nucl. Phys. A 378 (1982) 407-417; B. Gyarmati, A.T. Kruppa, Z. Papp, G. Wolf, Nucl. Phys. A 417 (1984) 393-404.

[7] J.A. Maruhn, W. Greiner, Z. Phys. 251 (1972) 431-457.

[8] K. Pruess, P. Lichtner, Nucl. Phys. A 291 (1977) 475-509.

[9] A.T. Kruppa, Z. Papp, Comp. Phys. Commun. 36 (1985) 59-78.

[10] A.C. Fonseca, J. Revai, A. Matveenko, Nucl. Phys. A 326 (1979) 182192; J. Revai, A. Matveenko, Nucl. Phys. A 339 (1980) 448-464.

[11] A. Diaz-Torres, Phys. Lett. B 594 (2004) 69-75.

[12] K. Hagino, T. Ichikawa, Phys. Rev. C 95 (2017) 054620-1-7.

[13] A. Diaz-Torres, Phys. Rev. C 74 (2006) 064601-1-13.

[14] A. Diaz-Torres, L.R. Gasques, M. Wiescher, Phys. Lett. B 652 (2007) $255-258$

[15] A. Diaz-Torres, Phys. Rev. Lett. 101 (2008) 122501-1-4.

[16] A. Diaz-Torres, in: Wolf Udo Schröder (Ed.), Nuclear Particle Correlations and Cluster Physics, World Scientific Publishing Co. Pte. Ltd., Singapore, 2017, pp. 447-470.

[17] W. Greiner, J.Y. Park, W. Scheid, Nuclear Molecules, first ed., World Scientific, Singapore, 1994. 